\title{
Mouse development hits the big screen
}

In recent decades, the combination of microscopy and fluorescent reporters has transformed our understanding of dynamic processes in cells and tissues. However, it remains challenging to image cells that are deep in tissues, are in photosensitive samples or are in tissues undergoing rapid morphological change, as happens during embryonic development.

Writing in Cell, Philipp Keller and colleagues ${ }^{1}$ present an improved light-sheet microscopy system that overcomes many of the difficulties associated with longterm imaging of live mammalian embryos. Using the approach, they generated the first movie of mouse embryonic development at single-cell resolution from gastrulation to early organogenesis.

In light-sheet microscopy, a whole plane of a specimen is illuminated simultaneously, enabling rapid imaging at high resolution and with minimal light exposure and phototoxicity - an approach that is ideally suited to sensitive samples. As with all optical techniques, the resolution and signalto-noise ratio of light-sheet microscopy are limited by scattering and autofluorescence and by continuous changes in optical properties induced by growth and reorganization of developing organisms.

Built on the AutoPilot technology developed for imaging of fish and fly embryos, which show less dramatic changes in size ${ }^{2}$, the improved microscope of Keller and colleagues ${ }^{1}$ tracks and adapts to changes in the embryo's optical properties, size and position, and it minimizes optical path length in the culture medium using custom objectives. This requires dynamic construction of an in silico reference model that automatically corrects for optical aberrations in different regions of the organism. All told, average resolution is improved 3.3-fold and signal strength 2.1-fold compared with the uncorrected version.

From the start of the authors' experiment, at embryonic day 6.5 , to its conclusion $48 \mathrm{~h}$ later, the volume of the embryo increases 250 -fold. Yet the authors are able to maintain

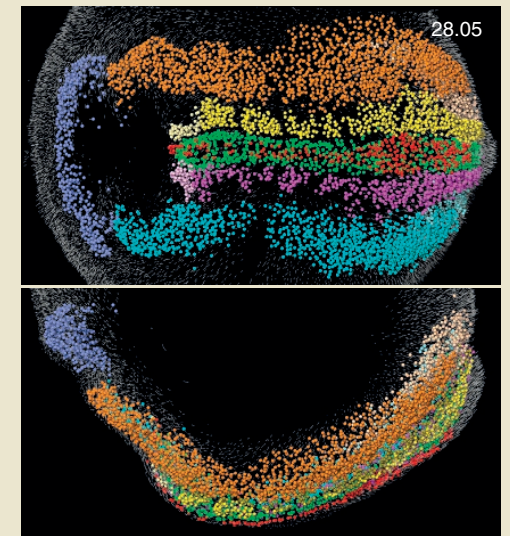

robust manner. The authors overcome this challenge with a method for mapping data from individual embryos to a common reference embryo. They use the method, called TARDIS, to construct a statistical fate map that shows the percentages of cells in an organ that derive from specific earlier populations. Such maps will be useful for selecting cell populations to study when trying to elucidate mechanisms in organ development. TARDIS also enables characterization of the coordinated movements of larger cell populations (morphodynamics) in a common framework. A complementary method presented in the work generates 'average' embryos that can facilitate analyses difficult to conduct with individual embryos, such as detecting differences between mutants.

Combining their new methods, the authors characterize the distribution and orientations of cell divisions along the developing neural tube and show that the orientation of cell division undergoes a marked change as neural tube closure progresses. Whereas cell divisions are mainly oriented along the rostral-caudal axis before neural tube closure, the orientations become more evenly distributed as tube closure progresses and finally tend to occur along the mediolateral axis as closure nears completion.

Keller and colleagues ${ }^{1}$ have shown that high-resolution imaging of complex developmental processes in mammalian embryos is feasible. The work also instructs as to how to develop image analysis software that extracts quantitative information from vast amounts of data. Detailed microscopy data such as those collected here will be invaluable for understanding both normal and aberrant development in mammals.

\section{Markus Elsner, Senior Editor}

Even at this early stage of embryogenesis, embryos at a given time point vary considerably in size, shape and stage of organogenesis, which makes it difficult to compare cellular dynamics in a quantitative and statistically
1. McDole, K. et al. Cell 175, 859-876.e33 (2018).

2. Royer, L.A. et al. Nat. Biotechnol. 34, 1267-1278 (2016).

3. Amat, F. et al. Nat. Methods 11, 951-958 (2014). 